\title{
द R Rearerch Suare \\ Characterization of Tn7-like transposons and its related antibiotic resistance among Enterobacteriaceae from livestock and poultry in China
}

Juan He

Sichuan University

Cui Li

Sichuan University

Pengfei Cui

Sichuan University

Hongning Wang ( $\square$ whongning@163.com )

Key Laboratory of Bio-Resource and Eco-Environment of Ministry of Education, College of Life Sciences, Sichuan

University;Animal Disease Prevention and Food Safety Key Laboratory of Sichuan Province;

\section{Research}

Keywords: Tn7-like, Enterobacteriaceae, antibiotic resistance, class 2 integrons, farms, Tn6765

Posted Date: April 8th, 2020

DOI: https://doi.org/10.21203/rs.3.rs-20496/v1

License: (9) (1) This work is licensed under a Creative Commons Attribution 4.0 International License. Read Full License 


\section{Abstract}

Background: This study was aimed to investigate the prevalence and structure of Tn7-like in Enterobacteriaceae from livestock and poultry as well as their possible role as reservoir of antibiotic resistance genes (ARGs).

Methods: Polymerase chain reaction (PCR) and DNA sequencing analyses were used for the characterization of Tn7-like, associated integrons and ARGs. The antimicrobial resistance profile of the isolates was examined by using disc diffusion test.

Results: Three hundred and seventy-eight Tn7-like-positive strains of Enterobacteriaceae were isolated, and included E. coli (128), Proteus(150), K. pneumonia(17), Salmonella(13), M. morganii (21) and A. baumannii(1), wherein high resistance was observed for Trimethoprim/Sulfamethoxazole and Streptomycin, and fifty percent of the strains were multidrug-resistant. Integrons class 2 were detected in all of the isolates and there are high frequency mutation sites especially in 535, a stop mutation. Variable region of class 2 integrons carried same gene cassettes, namely aadA1-sat2-dfrA1. From the 378 isolated strains, we found a new type of Tn7-like on a plasmid, named Tn6765.

Conclusions: These findings proved that the Tn7-like can contribute to the horizontal transmission of antibiotic resistant genes in livestock and poultry. As potential vessels for antibiotic resistance genes (ARGs), Tn7-like could not be ignored due to their efficient transfer ability in environments.

\section{Background}

Enterobacteriaceae include a large group of gram-negative bacillus with similar biological characteristics, They are widely distributed in the natural environment and the intestinal tract of humans and animals. As an opportunistic pathogen, Enterobacteriaceae is associated with urinary tract infection (1), nosocomial infection (2), and food poisoning (3). The widespread and irrational use of antibiotics in humans and animals has given rise to numerous multidrug-resistant Enterobacteriaceae (MRE) via the dissemination of antimicrobial resistance genes (AMRg) (4). This multiresistance may be mediated by chromosomally located resistance determinants or mutations in a resident gene. however, it may also develop through the acquisition of resistance genes or an array of resistance genes by horizontal transfer. This latter phenomenon is currently thought to play an increasing role in the development of multidrug resistance in Enterobacteriaceae (5). These resistance determinants of Enterobacteriaceae are generally disseminated via mobile elements (integrons, transposons and plasmids) in bacterial populations from animals and humans (4). One significant finding over the past 10 years is the appreciation of the dissemination of Tn7, and related elements called Tn7-like elements that contain homologs of the Tn7 transposition proteins, in highly diverged bacteria adapted to a remarkable number of different environments (6-8).

Tn7 is typically large, often $14 \mathrm{~kb}$ in size (9). However, comparative analysis of some derivatives of the Tn7, Tn7-like, shows that these elements are very diverse in terms of genetic structure and have accumulated several accessory resistance regions $(8,10)$. Tn7-like carry the determinants for resistance not only to heavy metals such as mercuric ions, copper, silver ions, tellurite, arsenate and arsenite, but also to antibiotics such as $\beta$-lactams (including carbapenems), quinolones, aminoglycosides, tetracyclines, amphenicols, trimethoprim, sulfamethoxazole, streptomycin and fosfomycin (11). The Tn7-like, as an important mobile genetic element of transposons, transfers various resistance genes between bacteria through its transposase, promotes the horizontal spread of drug resistance in bacteria.

The sequences of the two ends of the Tn7 transposon are highly conserved, which are respectively transposition module and class 2 integron system. Transposition module encodes five proteins required for two transposition path-ways, $\operatorname{tns} A, \operatorname{tns} B, \operatorname{tn} s C$, $\operatorname{tns} D$, and tnsE (tnsABCDE) (12). The basic transposition machinery of $\mathrm{Tn} 7$ and how it directs transposition into the attTn7 site is well understood and has been the subject of multiple reviews $(13,14)$. tns $A B C$ are required for both $\mathrm{Tn} 7$ transposition pathways, but only allow transposition when they function with one of two target site selecting proteins, $\operatorname{tns} D$ or $\operatorname{tns} E(15,16)$. Transposition with $t n s A B C+t n s D$ directs transposition into the single attTn7 site located downstream of $g / m S(15)$. Transposition with $t n s A B C+t n s E$ occurs preferentially into mobile plasmids through the ability of the tnsE protein to recognize features found enriched during DNA replication on the lagging-strand template $(17,18)$. 
The class 2 integron of Tn7 has an organization similar to that of the class I integron and carries three resistance gene cassettesaadA1, sat, and $d f r A 1$ (19-21)-close to an open reading frame, Int/2*. Int/2* has premature translation termination due to mutation of base 535 encoding integrase from $\mathrm{C}$ to $\mathrm{T}$, and has no function. Although these gene cassettes are fixed in Tn7 due to mutations in the homologous recombinase, they can be rearranged in hosts expressing the relevant recombinase, resulting in other combinations of antibiotic resistance genes (22).

However, there is a paucity of data on the prevalence and role of Tn7-like transposons in antibiotic resistant Enterobacteriaceae in many countries such as china especially from livestock and poultry. The aim of this study was to determine the frequency of Tn7like transposons and its associated integrons in Enterobacteriaceae which from swine and chicken, and to investigate the association between the presence of Tn7-like and antibiotic resistance patterns, so as to better understand the characteristics of Tn7-like and resistant Enterobacteriaceae in farming animals. Additionally, we plan to analyze the skeletal structure of our Tn7-like and compared it with other Tn7-like to obtain insight into the horizontal transfer of resistance genes and the diversity and evolution of Tn7-like.

\section{Methods}

\section{Bacterial isolates}

A total of 1482 consecutive and unduplicated clinical isolates of Enterobacteriaceae were collected from livestock and poultry in China from 2018 to January 2020. The isolates were from the intestines of dead animals, feces, cloacal swab and drinking water (Table 1). All the isolates were presumptively identified through phenotypic methods, including colony morphology on MacConkey Agar or Eosin-Methylene Blue Agar. And the identification of these isolates was later confirmed using 16S rRNA gene sequencing. All Enterobacteriaceae strains were stored at $-20^{\circ} \mathrm{C}$ in $50 \%$ glycerol. The standard strain of Escherichia coli (ATCC25922) was used as a susceptibility control. Rifampin-resistant strain $E$. coli600 was used as the recipient strain in the conjugation experiments.

Table 1

Bacterial Strains Isolated from 2018 to 2020

\begin{tabular}{|llll|}
\hline Source & Species & No. of strains & Note \\
\hline Pigs & Escherichia coli & 413 & Fecal sample, cloacal swab , drinking \\
\hline & Proteus mirabilis & 208 & water, or small intestine of dead \\
& Providencia & 164 & swine from 12 pig farms in 6 \\
\hline & Morganella morganii & 146 & different provinces of China. \\
\hline & Klebsiella pneumoniae & 141 & \\
\hline Sotal & Salmonella enterica & 82 & \\
\hline Chicken & Escherichia coli & 121 & Fecal sample, cloacal swab , drinking \\
\hline & Proteus mirabilis & 53 & water, or small intestine of dead \\
\hline & Providencia & 46 & chicken from 10 poultry farms in 5 \\
\hline Klebsiella pneumoniae & 24 & different provinces of China. \\
\hline & Salmonella enterica & 76 & \\
\hline
\end{tabular}




\section{Detection Of Tn7-like Transposons By Polymerase Chain Reaction (PCR)}

Tn7-like transposons were tested in 1483 Enterobacteriaceae isolates using PCR targeting tns $A$, a transposase-encoding gene. The isolates were grown overnight (18-24 h) in Brain Heart Infusion(BHI) broth (Oxoid, UK) at $37^{\circ} \mathrm{C}$ with a rotation speed of $200 \mathrm{rpm}$, and the DNA template was prepared using the boiling method (23). The PCR mixture was prepared with a final volume of $20 \mu l$, containing $1 \mu \mathrm{l}$ of template DNA, $8 \mu \mathrm{lddH_{2 }} 0,10 \mu \mathrm{l} \mathrm{Taq} \mathrm{PCR} \mathrm{MasterMix} \mathrm{and} 0.5 \mu \mathrm{l}$ each primer. The specific primers for detecting tnsA gene are shown in Table S1. The amplified PCR products were separated by electrophoresis in $1.2 \%$ agarose gels and visualised after staining with Goldview dye.

\section{Antibiotic Sensitivity Test}

The antibiotic resistance profile of all Tn7-like positive isolates was determined using the disc diffusion method, as per the Clinical and Laboratory Standards Institute standards (56). Briefly, Mueller-Hinton agar was used as the test medium for each of the following antimicrobials (all discs from Oxoid, Basingstoke, UK): Ampicillin (AMP, $10 \mu \mathrm{g}$ ), Imipenem(IPM, $10 \mu \mathrm{g}$ ), Cefoxitin (FOX, $30 \mu \mathrm{g})$, Ciprofloxacin (CIP, $5 \mu \mathrm{g})$, Streptomycin(S, $10 \mu \mathrm{g})$, Gentamicin(CN,10 $\mu \mathrm{g})$, Florfenicol(FFC, $30 \mu \mathrm{g})$, Enrofloxacin(ENR, $5 \mu \mathrm{g})$, Tetracycline(TGC, $30 \mu \mathrm{g})$, Aztreonam(ATM, $30 \mu \mathrm{g})$, Ceftazidime(CAZ, $30 \mu \mathrm{g})$ and Trimethoprim/Sulfamethoxazole $(\mathrm{SXT}, 1.25 / 23.75 \mu \mathrm{g})$. E. coli ATCC 25922 was used as a quality control strain.

\section{Characterization Of The Integrons And Associated ARGs}

The isolated Tn7-like Enterobacteriaceae strains were checked for the presence of int/2 integrase genes by PCR, using primers(Table S1) and methodology as previously described by $(24,25)$, and these mutation site sequence of int/2 were visualized with Weblogo (http://weblogo.berkeley.edu/logo.cgi). Subsequently, strains harboring int/2 genes were elaborated for the amplification of its three resistance gene cassettes (aadA1, sat2 and dfrA1). For this purpose, primer pairs which are listed in Table $\mathrm{S} 1$, were used to amplify the gene cassette by targeting aadA1, sat2 and $d f r A 1$, respectively.

\section{WGS And Analysis}

We also analysed genetic environment of Tn7-like transposons among strains exhibiting unique resistance phenotypes. The whole genome of the Tn7-like-positive strain was sequenced using Illumina MiSeq with a 200-fold sequencing depth and the nanopore platform with a 100-fold sequencing depth. Genome assembly was carried out by de novo assembly in SMRT portal version 3.2.0, and the sequence was annotated using the NCBI Prokaryotic Genome Annotation Pipeline v4.2. Mobile elements, resistance genes, and other features were annotated by INTEGRALL (26), ISfinder (27), ResFinder (28), PlasmidFinder (29), and the Tn Number Registry (30) online databases, and the analysis was conducted using the BLAST program (http://blast.ncbi.nlm.nih.gov/Blast.cgi).

\section{Horizontal Transfer Of Tn7-like Transposon}

Conjugation was performed using rifampin-resistant E. coli EC600 as the recipient and the P. mirabilis SCBX1.1 isolate as the donor with selection on Salmonella Shigella agar plates containing $20 \mathrm{mg} /$ liter rifampin and $8 \mathrm{mg} /$ liter florfenicol. Successful horizontal transfer of plasmid p1.1.2 containing Tn7-like transposon was confirmed using antibiotic sensitivity test and PCR as mentioned above, then the conjugation frequency was calculated as transconjugants divided by number of donors (31). The stability of p1.1.2 in the positive transconjugants (designated EC-600-Pp1.1.2) was determined by passage in Brain Heart Infusion(BHI) broth lacking antibiotics as described previously (32).

\section{Statistical analysis}

Variables are expressed as percentages (\%). Comparison of incidence of Tn7-like in Enterobacteriaceae isolates versus Tn7-like lacking isolates was assessed using the Chi square test. Statistical analysis was conducted with the GraphPad Prism 8 software. 
$P$ value of $<0.05$ was considered as significant.

\section{Genbank Accession Number}

The complete sequences of p1.1.1 (CP047113), p1.1.2 (CP047114), and all Tn7-like; Tn6763 (MN641830), Tn6764 (MN628641), and Tn6765 (CP047113), identified in the present study were submitted to NCBI GenBank.

\section{Results}

\section{Prevalence of Tn7-like transposon in Enterobacteriaceae}

A total of 1482 strains of Enterobacteriaceae were isolated during the period 2018.6-2020.1 through analysis of samples collected from Sichuan, Hainan, Chongqing, Shandong, Hebei and Anhui provinces. Of the 1482 Enterobacteriaceae strains examined, 378 (25.5\%) contained Tn7-like transposon. They included 128(8.6\%) Escherichia coli, 150(10.1\%) Proteus, 48(3.2\%) Providencia, $21(1.4 \%)$ Morganella morganii, 17(1.1\%) Klebsiella pneumoniae, $13(0.9 \%)$ Salmonella enterica and $1(0.06 \%)$ Acinetobacter baumannii (Fig. 1). The positive rate of Tn7-like transposon in proteus was significantly higher than that of other strains $(p<$ 0.0001).

\section{Antimicrobial Resistance Phenotypes}

A total of $378 \mathrm{Tn} 7$-like positive isolates were examined for their resistance against 10 antibiotics (Table 2). Two hundred and fourteen $(56.61 \%)$ isolates showed resistance to three or more antibiotic classes and were considered as multidrug-resistance (MDR). Except for Acinetobacter baumannii, Proteus and Salmonella have the highest multidrug resistance rates of $65.69 \%$ and $63.90 \%$, respectively. Notably, high resistance rates were observed for Streptomycin (88.7\%) and Trimethoprim/Sulfamethoxazole (74.6\%), followed by Ampicillin (63.23\%). And low resistance rates to Ceftazidime (5.82\%), Cefoxitin (5.82\%) and Aztreonam (4.23\%) were detected. Most of the Enterobacteriaceae isolates were highly susceptible to Imipenem.

Table 2

Rates of Resistance to Antimicrobial Agents of Tn7-like Positive Isolates.

\begin{tabular}{|c|c|c|c|c|c|c|c|c|}
\hline $\begin{array}{l}\text { Antimicrobial } \\
\text { agent }\end{array}$ & $\begin{array}{l}\text { E.coli } \\
(n= \\
128), \\
\%\end{array}$ & $\begin{array}{l}\text { Proteus } \\
(n= \\
150), \\
\%\end{array}$ & $\begin{array}{l}\text { Providencia } \\
(n=48) \\
\%\end{array}$ & $\begin{array}{l}\text { M.morganii } \\
(n=21) \\
\%\end{array}$ & $\begin{array}{l}\text { K.pneumoniae } \\
(n=17) \\
\%\end{array}$ & $\begin{array}{l}\text { S.enterica } \\
(n=13), \\
\%\end{array}$ & $\begin{array}{l}\text { A.baumannii } \\
(n=1) \\
\%\end{array}$ & $\begin{array}{l}\text { Total } \\
(\mathrm{n}= \\
378), \\
\%\end{array}$ \\
\hline $\mathrm{CN}(10 \mu \mathrm{g})$ & 24.22 & 30.67 & 12.50 & 9.52 & 11.76 & 38.46 & 0.00 & 24.34 \\
\hline $\mathrm{S}(10 \mu \mathrm{g})$ & 84.38 & 90.00 & 95.83 & 90.48 & 70.59 & 84.62 & 100.00 & 87.83 \\
\hline TGC $(30 \mu \mathrm{g})$ & 56.25 & 32.67 & 16.67 & 4.76 & 47.06 & 69.23 & 100.00 & 39.15 \\
\hline CAZ $(30 \mu \mathrm{g})$ & 6.25 & 3.33 & 8.33 & 0.00 & 0.00 & 38.46 & 0.00 & 5.82 \\
\hline IPM $(10 \mu g)$ & 1.56 & 0.00 & 0.00 & 0.00 & 0.00 & 0.00 & 0.00 & 0.53 \\
\hline FOX $(30 \mu \mathrm{g})$ & 7.03 & 6.00 & 2.08 & 0.00 & 11.76 & 7.69 & 0.00 & 5.82 \\
\hline $\operatorname{SXT}(1.25 / 23.75 \mu \mathrm{g})$ & 67.97 & 85.33 & 75.00 & 52.38 & 41.18 & 76.92 & 100.00 & 74.07 \\
\hline ATM $(30 \mu \mathrm{g})$ & 3.91 & 1.33 & 4.17 & 0.00 & 5.88 & 46.15 & 0.00 & 4.23 \\
\hline CIP $(5 \mu g)$ & 22.66 & 28.00 & 16.67 & 4.76 & 0.00 & 23.08 & 0.00 & 21.96 \\
\hline AMP $(10 \mu \mathrm{g})$ & 60.94 & 70.67 & 58.33 & 52.38 & 35.29 & 69.23 & 100.0 & 63.23 \\
\hline MDR & 55.22 & 65.96 & 52.25 & 29.93 & 22.83 & 63.90 & 100.0 & 56.61 \\
\hline
\end{tabular}




\section{Prevalence And Cassette Gene Of Tn7-like Integrons}

The positive rate of class 2 integrons in 378 Enterobacteriaceae strains was 100\%. Through sequencing and sequence comparison, the results showed that int/2 usually had mutation sites, and most of them were at 349,372,379,535,617,767 and 774(Fig. 2). On amplification, 347 isolates were found to carry the resistance gene cassette (aadA1, sat2 and dfrA7) related to int/2, whereas 31 strains had different aadA1, sat2 and $d f r A 1$ gene deletions, respectively. 24 isolates were missing one gene cassette, and 7 isolates were missing two gene cassette. A single S.enterica only harbored $d f r A 1$ (Table 3).

Table 3

Cassette Gene of Tn7-like Integrons.

\begin{tabular}{|c|c|}
\hline No. of isolates & Cassette gene \\
\hline 121(E.coli $), 142$ (Proteus),41(Providencia),19(M.morganii) $15($ K.pneumoniae),8 (S.enterica),1(A.baumannii) & aadA1,sat2,dfrA1 \\
\hline 2(E.coli), 3(Proteus), 1(Providencia),2(S.enterica) & aadA1,sat2 \\
\hline 2(E.coli), 5(Proteus), 1(Providencia),2(M.morganii),2 (K.pneumoniae) & aadA1,dfrA1 \\
\hline 1(E.coli),1(Providencia), 2(S.enterica) & sat2,dfrA1 \\
\hline 1(E.coli $), 1$ (Providencia), & aadA1 \\
\hline 1(E.coli),3(Providencia), & sat2 \\
\hline 1(S.enterica) & dfrA1 \\
\hline
\end{tabular}

\section{Structural Analysis Of Tn7-like}

Among the Tn7-like-carrying strains, three multi-drug resistant bacteria were randomly selected for whole-genome sequencing, and yielded three complete Tn7-like structures. According to the transposon nomenclature (http://transposon.lstmed.ac.uk/), we designated them Tn6763, Tn6764, and Tn6765, respectively. Tn6763 (GenBank accession number MN641830) and Tn6764 (GenBank accession number MN628641) were located on the chromosomes of Proteus and E. coli, respectively. They are typical Tn7 transposons comprising the transposition module (tnsA, tnsB, tnsC, tnsD and tnsE), three gene cassettes (aadA1, sat2 and $d f r A 1)$, and an inactive class 2 integrase gene. Besides, both of them inserted an insB-1 next to the aadA1 gene (Fig. 3).

Tn6765 is located on a plasmid of Proteus mirabilis SCBX1.1, named p1.1.2 (GenBank accession number CP047114). Sequence analysis showed that 19 resistance genes, except for $c f$, were carried by the Tn7-like transposon (Fig. 3).The novel MDR transposon harboring 18 different resistance genes. including blaDHA-1 (cephalosporin resistance), qnrA1 (fluoroquinolone), $\operatorname{aac}(6=)$-lb-cr. (fluoroquinolone and aminoglycosides), floR (chloramphenicol/florfenicol), $\mathrm{mphE}$ and $\mathrm{msrE}$ (macrolide), and lunF (lincosamide) genes. While the $c f r$ gene is carried by another 12795 bp plasmid p1.1.1(GenBank accession number CP047113), which exists in the same strain as p1.1.2. Tn6765 is $64752 \mathrm{bp}$ (corresponding to bases 47309 to 112060 in GenBank accession number CP047114) with an average GC content of 52.94\% that differs from that of the rest of the $P$. mirabilis plasmid (GC content, 44.35\%). It has partial characteristics of the Tn7 transposon (Fig. 3), which contained transposase genes $\operatorname{tns} A$, tns $B$ and $\operatorname{tns} C$. But its transposition module lost $t n s E$ gene and the $t n s D$ was truncated by insertion of the inverted repeat (IR mcp ) of Tn1721.

Tn6765 was highly homologous with Tn6450 located on the chromosome detected by Chen (33). The similarity of nucleic acid sequence was more than $99 \%$, and the coverage was $96 \%$. The differences between the two are mainly in the following four aspects: (1) Tn6765 has a hypotheical protein gene before blaDHA-1; (2) The ampR gene of Tn6450 was replaced by lysR and HypA in Tn6765; (3) An incomplete aac(3)-IV was inserted between ISEc59 and aph(4)-la; (4) Tn6450 is inserted downstream of the glmS gene, encoding glucosamine 6-phosphate synthase and surrounded by 5-bp direct repeats (CCAAT), Whereas Tn6765 is located on the plasmid, there is no glms gene and repeated sequences at both ends(Figs. 3 and 4). The plasmid carrying Tn6765 was $138818 \mathrm{bp}$ in size and had a GC content of $44.35 \%$, which was named as p1.1.2. Blastn results showed that, except for the Tn6765 Tn7-like MDR region, other regions of plasmid p1.1.2. showed $99.98 \%$ similarity and $91 \%$ coverage with nucleic acid 
sequence of P.mirabilis plasmid pPm60 (GenBank accession number MG516911) that also carried the Tn7-like transposon published in NCBI database (Fig. 4).

\section{Horizontal Transfer Of Tn7-like}

Double-antibiotics Salmonella Shigella agar plates (300ug / $\mathrm{ml}$ rifampicin $+10.24 \mathrm{ug} / \mathrm{ml}$ flufenicol) were used to screen the transconjugant. The results of drug sensitivity, electrophoresis, and sequencing showed that the plasmid p1.1.2 could be successfully transferred to E. coli EC600 (Table 4). A novel Tn7-like (Tn6765) transconjugant was obtained through the conjugative transfer test, and at a frequency of $1.5 \times 10^{-1}$ transconjugants per donor (average of three independent determinations). The stability of Tn6765 was determined by continuous passage propagation lasting 10 days (20 passages) in the absence of antimicrobial pressure. One hundred clones in the 21st passage were picked to detect the presence of Tn6765. The results of all PCRs were positive. Showed that Tn6765 can be stably inherited in the recipient bacteria.

Table 4

Antimicrobial resistance phenotype and inhibitory zone diameter of Proteus mirabilis SCBX1.1 isolate, Escherichia coli EC600 and EC600 with plasmid P1.1.2

\begin{tabular}{|c|c|c|c|c|c|c|}
\hline \multirow[t]{2}{*}{ Antibiotics } & \multicolumn{3}{|c|}{ Inhibitory zone diameter(mm) } & \multicolumn{3}{|c|}{ Antimicrobial resistance phenotype } \\
\hline & SCBX1.1 & EC600 & $\mathrm{EC} 600+\mathrm{P} 1.1 .2$ & SCBX1.1 & EC600 & EC600 + P1.1.2 \\
\hline $\mathrm{CN}(10 \mu \mathrm{g})$ & 9 & 24 & 8 & $\mathrm{R}$ & S & $\mathrm{R}$ \\
\hline $\mathrm{S}(10 \mu \mathrm{g})$ & 0 & 27 & 0 & $\mathrm{R}$ & S & $\mathrm{R}$ \\
\hline $\mathrm{FFC}(30 \mu \mathrm{g})$ & 0 & 25 & 0 & $\mathrm{R}$ & S & $\mathrm{R}$ \\
\hline $\mathrm{CAZ}(30 \mu \mathrm{g})$ & 13 & 30 & 11 & $\mathrm{R}$ & S & $\mathrm{R}$ \\
\hline $\operatorname{ENR}(5 \mu \mathrm{g})$ & 0 & 28 & 9 & $\mathrm{R}$ & $\mathrm{S}$ & $\mathrm{R}$ \\
\hline FOX $(30 \mu \mathrm{g})$ & 13 & 30 & 0 & $\mathrm{R}$ & S & $\mathrm{R}$ \\
\hline $\operatorname{SXT}(1.25 / 23.75 \mu \mathrm{g})$ & 0 & 31 & 0 & $\mathrm{R}$ & S & $\mathrm{R}$ \\
\hline $\mathrm{CIP}(5 \mu \mathrm{g})$ & 11 & 27 & 0 & $\mathrm{R}$ & $\mathrm{S}$ & $\mathrm{R}$ \\
\hline AMP $(10 \mu \mathrm{g})$ & 0 & 22 & 0 & $\mathrm{R}$ & $\mathrm{S}$ & $\mathrm{R}$ \\
\hline
\end{tabular}

\section{Discussion}

Laboratory experiments have shown that $\mathrm{Tn} 7$ and its derivatives are capable of transposition in many different hosts, but few naturally occurring examples have been isolated (7) In this study, we examined the occurrence and prevalence of Tn7-like in Enterobacteriaceae isolates obtained from several farms of chicken and swine raised for meat purpose. Of 7 Enterobacteriaceae species in the collection, the most prevalent species included Proteus (150 isolates, 39.7\%) and Escherichia coli (128 isolates, $33.9 \%)$. One hundred isolates (26.5\%) were assigned to other Enterobacteriaceae species. Statistical analysis showed that the presence of Tn7-like was different among different bacteria genera $(P<0.0001)$ (Fig. 1). The result indicated Proteus malleable genomes contribute to facilitate high-frequency insertion of transposons like Tn7-like, which was similar to the findings of other studies $(31,34,35)$. Also, Escherichia coli is the representative strain of the genus Escherichia, which is the abundant type of bacteria in the intestine of humans and animals (36), so its proportion in Tn7-like positive Enterobacteriaceae is also large. Our results provide evidence of the pigs and chickens as a possible reservoir of Tn7-like carrying strains with the antibiotic resistance gene. Enterobacteriaceae, as a widespread intestinal bacterium, its carriage Tn7-like are likely to have a serious impact on human life through animals such as pigs and chickens, and is a risk that deserves our attention. 
In multidrug-resistant Enterobacteriaceae bacteria, Tn7-like transposons play a very vital role due to their high capability for transferring antimicrobial resistance genes (37). Through drug susceptibility tests, we found that the multi-drug resistance of Enterobacteriaceae carrying Tn7-like is widespread in various strains. Except for M.morganii and K.pneumoniae, the multi-drug resistance rates of other strains are above 50\%. Among them, Proteus (65.69\%) and Salmonella (63.90\%) are more prominent(Table 2). Proteus is an important drug-resistant storage bacteria because of its highly efficient genetic structure. It is usually considered commensal in the gut, but it can also cause food poisoning and is most commonly recognized clinically as a cause of urinary tract infections $(38,39)$. Salmonella, one of the most common and widely distributed foodborne pathogenic microorganisms, is a major cause of food-borne salmonellosis $(40,41)$. And the food-borne salmonellosis is typically acquired through consumption of contaminated poultry meat and eggs (42). Obviously, for both Proteus and Salmonella, carriage of Tn7like, each containing a set of resistance genes, may increases the chances of horizontal transfer of multiple resistance determinants to susceptible strains, and may in turn confer unique advantages to the host and enable them survive a strong antimicrobial selection pressure especially in poultry and livestock farm settings.

Tn7-like isolates are particularly resistant to Trimethoprim/Sulfamethoxazole and Streptomycin because of the int/2-associated

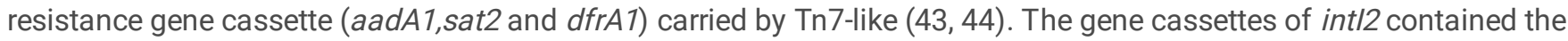
aminoglycoside adenyltransferase ( $\operatorname{aad} A 1$ and $\operatorname{aad} A 2)$, dihydrofolate reductase (dfrA1), and streptothricin acetyltransferase (sat2) encoding genes, which are responsible for streptomycin-spectinomycin, trimethoprim, and streptothricin resistance, respectively area (45). In this study, we used the PCR method to determined the occurrence of class 2 integrons and associated gene cassettes ( aadA1, sat2 and dfrA1) in Tn7-like-positive isolates. Similar gene cassettes (aadA1, sat2, and dfrA1)have been obtained in the most of animals-derived Enterobacteriaceae isolates $(46,47)$. The presence of the gene cassette and its corresponding phenotypic resistance was matched(Tables 2 and 3). This explains why Tn7-like bacteria are highly resistant to Trimethoprim/Sulfamethoxazole and Streptomycin, and may pose the risk of widespread dissemination of large, single assemblage repositories of resistance genes and threatening the emergence of a post-antibiotic era (48). The high prevalence of the Tn7-like containing antibiotic resistance genes detected in the current study could be attributed to extensive use of antibiotics for disease prevention, treatment and growth enhancement in farm animals in the studied. Specifically, spectinomycin is widely used against the gonorrhea and respiratory/enteric infections in humans and animals (25). Tn7-like have been so successful at spreading into diverse relevant taxa that they could be used as a proxy for anthropogenic pollution $(49,50)$.

The int/2 downstream of Tn7-like transposons mediate the deletion of gene cassettes (aadA1,sat2, and dfrA1).Through sequencing, we found that there were multiple mutated sites in int12, among which 535 base mutations (C mutated to T) were terminating mutations(Fig. 2), which led to premature termination of integrase gene translation, making its gene cassette sequence show a high degree of stability, usually aadA1, sat2 and $d f r A 1$ (51). Karin Hansson et al. changed the stop codon 179 of the intl2 gene into the codon encoding glutamic acid $(\mathrm{E})$, and found that int/2*179E promoted the specific excision of each gene cassettes in $\mathrm{Tn} 7$ at different frequencies (22). Some of the gene cassettes deletions investigated in this study are precisely because the 535 base mutation produces a functional integrase gene int/2 (Fig. 2 and Table 3). We show here experimentally that the second class of integrase and associated gene cassettes in Tn7 can promote recombination between resistant gene present in the host, but only after changing the termination codon to a sense codon (22). This makes Tn7-like a better reservoir of antibiotic resistance.

The structure of the Tn7-like transposon is changeable and interrelated. We have obtained a new transposon Tn6765. By comparison analysis, we know that Tn6765 and Tn6450 have a very high similarity. Both are Tn7-like transposons, which are generated by inserting sequences and transposons carrying flanking drug resistance genes integrated into the Tn7 transposon variable region. Their transposable module was interrupted by the transposon $\operatorname{Tn} 1721$ at the transposase gene $\operatorname{tns} D$, and $\operatorname{Tn} 1721$ was interrupted by the downstream IS26 (33). The structures of Tn6765 and Tn6450 are complex and highly homologous, suggesting that they may have undergone similar evolutionary processes. Tn6765 carries five IS26, two ISCR1, one IS1353, one IS1006, one ISCR2, one ISAba1, one ISEC59, and 2 defective transposons Tn1721 and Tn21, indicating that this transposon is generated by multiple mobile genetic elements carrying lateral resistance genes integrated into the variable region. There are a few genetic differences between Tn6450 and Tn6765(Fig. 3). Noteworthily, Tn6450 is located on the chromosome of Proteus mirabilis but Tn6765 is located on the plasmid(Fig. 4), which confirmed that Tn7-like can be transmitted alternately on the chromosome and plasmid by cutting and inserting. Through the conjugative transfer test, Tn6765 was successfully transferred to EC600 with the plasmid. 410minously, like its distant relative Tn7, Tn6765 may exhibit the potential to jump bacterial species readily. To make 
matters worse, Tn6450 comes from a chicken source (33) and Tn6765 from a pig source, suggesting that Tn7-like can be transmitted from one animal to another with bacterial hosts.

Proteus SCBX1.1 carrying Tn6765 also contains another cfrplasmid P1.1.1 (Fig. 6). The cfr-containing segment (corresponding to bases 8925 to 12726 to 4624 in GenBank accession number CP047113) harbours a genetic structure (Fig. 5), showing homology to the cfr segment characterized in P. cibarius G11 (Genebank accession number CP047287), which is partially differ from the Chen et al. reported the existence of IS26-cfr-DTn554 tnpB-DTn3 family tnpA-IS26 section in another Tn7-like Tn6451 (52) (Fig. 5). Although the cfr section of Tn6765 and Tn6451 is slightly different, this also suggests an evolutionary direction of the Tn6765 carrying strain. By comparing the genetic structure of different Tn7-like transposons, we can speculate that the multidrug-resistant Tn7-like transposons have a certain evolutionary relationship, which confirmed that Tn7-like plays a vital role in the field of storing resistance genes.

Unlike the DNA blueprints of higher organisms, Enterobacteriaceae bacterial genomes exhibit an extraordinary degree of plasticity and intraspecies diversity (53). A key feature of Enterobacteriaceae is the constant mobility of various segments of the genome both with in a single cell and between cells. This mobility plays a significant role in the evolution of bacteria through tuning of genetic architecture and the lateral acquisition of new genes that may provide a survival advantage, thus further adapting bacteria to new environments (54). Transposons as the major contributors to the acquisition and dissemination of antibiotic resistance genes (ARGs), and Tn7 are their central agents (55). One Tn7 relative may operate as a "founder element" that can locate and safely transpose into the bacterial chromosome, bringing with it other mobile elements and possibly attachment sites for bacteriophages, integrons, or other transposons. After multiple Tn7-like transposition events accumulate in the attachment site, recombination between these elements may enhance evolution by reassortment (7).

\section{Conclusions}

To the best of our knowledge, this study is the first report showing the prevalence and baseline characteristics of Tn7-like in Enterobacteriaceae isolates from livestock and poultry in China. Our results showed that Tn7-like transposon has a great effect on bacterial resistance, and they can continuously accumulate ARGs in the evolutionary process to consolidate the bacteria's defense ability, which makes bacterial treatment more difficult. Animals are possible reservoirs and a source for the dissemination of Tn7like transposon associated ARGs in the environment. Multiple antibiotic resistance gene determinants in Tn7-like, especially when found in producing chickens and swine is highly worrisome and may become a serious threat by spreading associated resistance genes in other nearby animals and the humans connected therewith. Therefore, strict preventive measures should be taken to stop the spread and emergence of mobile genetic resistance determinants in food producing poultry and livestock in China and the world, and our study provides an important reference for this.

\section{Abbreviations}

PCR: polymerase chain reaction

MDR: multidrug resistance;

MRE: multidrug-resistant Enterobacteriaceae;

ARGs: antibiotic resistance genes;

AMRg: antimicrobial resistance genes ;

AMP: Ampicillin;

IPM: Imipenem;

FOX: Cefoxitin;

CIP: Ciprofloxacin; 
S: Streptomycin;

CN: Gentamicin;

FFC: Florfenicol;

ENR: Enrofloxacin;

TGC: Tetracycline;

ATM: Aztreonam;

CAZ: Ceftazidime;

SXT: Trimethoprim/Sulfamethoxazole;

E.coli: Escherichia coli;

M. morganii: Morganella morganii;

K.pneumoniae: Klebsiella pneumoniae;

S.enterica: Salmonella enterica;

A.baumannii: Acinetobacter baumannii

\section{Declarations}

\section{Acknowledgments}

The authors are grateful to Adam P. Roberts (University College London) for helpful suggestions regarding the nomenclature of Tn6763, Tn6764 and Tn6765.

\section{Funding}

This work was support by the National Natural Science Foundation of China (Grant No. 31830098) and the National Key Research and Development Program of China (Grant No. 2016YFD0501608).

\section{Availability of data and materials}

Please contact author for data requests.

\section{Authors' Contributions}

All authors listed, have made substantial, direct and intellectual contribution to the work, and approved it for publication. Juan He conceived the study and drafted the manuscript. Juan He and Cui Li collected samples and conducted data statistics. Juan He, Cui Li and Pengfei Cui performed experiments. Hongning Wang supervised the research.

\section{Ethics approval and consent to participate}

Not applicable in view of the nature of the study.

\section{Consent for publication}

Not applicable.

\section{Competing interests}


The authors declare that they have no competing interests.

\section{Author details}

${ }^{1}$ Animal Disease Prevention and Food Safety Key Laboratory of Sichuan Province, Key Laboratory of Bio-resources and Ecoenvironment, Ministry of Education, College of life science, Sichuan University, Chengdu, China

${ }^{2}$ Animal Disease Prevention and Food Safety Key Laboratory of Sichuan Province, Chengdu,

Sichuan, China.

\section{References}

1. Keepers TR, Gomez M, Celeri C, Krause KM, Biek D, Critchley I. Fosfomycin and Comparator Activity Against Select Enterobacteriaceae, Pseudomonas, and Enterococcus Urinary Tract Infection Isolates from the United States in 2012. Infectious diseases therapy. 2017;6(2):233-43.

2. Amin AK, Wareham DW. Plasmid-mediated quinolone resistance genes in Enterobacteriaceae isolates associated with community and nosocomial urinary tract infection in East London, UK. Int J Antimicrob Agents. 2009;34(5):490-1.

3. Gelbicova T, Barakova A, Florianova M, Jamborova I, Zelendova M, Pospisilova L, et al. Dissemination and Comparison of Genetic Determinants of mcr-Mediated Colistin Resistance in Enterobacteriaceae via Retailed Raw Meat Products. Frontiers in microbiology. 2019;10:2824.

4. Rehman MU, Zhang H, lqbal MK, Mehmood K, Huang S, Nabi F, et al. Antibiotic resistance, serogroups, virulence genes, and phylogenetic groups of Escherichia coli isolated from yaks with diarrhea in Qinghai Plateau, China. Gut pathogens. 2017;9:24.

5. Leverstein-van Hall MA, Box AT, Blok HE, Paauw A, Fluit AC, Verhoef J. Evidence of extensive interspecies transfer of integronmediated antimicrobial resistance genes among multidrug-resistant Enterobacteriaceae in a clinical setting. The Journal of infectious diseases. 2002;186(1):49-56.

6. Mitra R, McKenzie GJ, Yi L, Lee CA, Craig NL. Characterization of the TnsD-attTn7 complex that promotes site-specific insertion of Tn7. Mobile DNA. 2010;1(1):18.

7. Parks AR, Peters JE. Transposon Tn7 is widespread in diverse bacteria and forms genomic islands. J Bacteriol. 2007;189(5):2170-3.

8. Parks AR, Peters JE. Tn7 elements: engendering diversity from chromosomes to episomes. Plasmid. 2009;61(1):1-14.

9. Peters JE. Tn7. Microbiology spectrum. 2014;2(5).

10. Peters JE, Fricker AD, Kapili BJ, Petassi MT. Heteromeric transposase elements: generators of genomic islands across diverse bacteria. Mol Microbiol. 2014;93(6):1084-92.

11. Liang Q, Yin Z, Zhao Y, Liang L, Feng J, Zhan Z, et al. Sequencing and comparative genomics analysis of the IncHI2 plasmids pT5282-mphA and p112298-catA and the IncHI5 plasmid pYNKP001-dfrA. Int J Antimicrob Agents. 2017;49(6):709-18.

12. Peters JE, Craig NL. Tn7: smarter than we thought. Nature reviews Molecular cell biology. 2001;2(11):806-14.

13. Fricker AD, Peters JE. Vulnerabilities on the lagging-strand template: opportunities for mobile elements. Annual review of genetics. 2014;48:167-86.

14. Peters JE. Targeted transposition with Tn7 elements: safe sites, mobile plasmids, CRISPR/Cas and beyond. Mol Microbiol. 2019;112(6):1635-44.

15. Waddell CS, Craig NL. Tn7 transposition: two transposition pathways directed by five Tn7-encoded genes. Genes Dev. 1988;2(2):137-49.

16. Waddell CS, Craig NL. Tn7 transposition: recognition of the attTn7 target sequence. Proc Natl Acad Sci USA. 1989;86(11):3958-62.

17. Peters JE, Craig NL. Tn7 recognizes transposition target structures associated with DNA replication using the DNA-binding protein TnsE. Genes Dev. 2001;15(6):737-47. 
18. Parks AR, Li Z, Shi Q, Owens RM, Jin MM, Peters JE. Transposition into replicating DNA occurs through interaction with the processivity factor. Cell. 2009;138(4):685-95.

19. Sundstrom L, Radstrom P, Swedberg G, Skold O. Site-specific recombination promotes linkage between trimethoprim- and sulfonamide resistance genes. Sequence characterization of $\mathrm{dhfrV}$ and sull and a recombination active locus of Tn21. Molecular general genetics: MGG. 1988;213(2-3):191-201.

20. Sundstrom L, Roy PH, Skold O. Site-specific insertion of three structural gene cassettes in transposon Tn7. J Bacteriol. 1991;173(9):3025-8.

21. Sundstrom L, Skold 0 . The dhfrl trimethoprim resistance gene of $\mathrm{Tn} 7$ can be found at specific sites in other genetic surroundings. Antimicrob Agents Chemother. 1990;34(4):642-50.

22. Hansson K, Sundstrom L, Pelletier A, Roy PH. Intl2 integron integrase in Tn7. J Bacteriol. 2002;184(6):1712-21.

23. Teh CS, Chua KH, Puthucheary SD, Thong KL. Further evaluation of a multiplex PCR for differentiation of Salmonella paratyphi A from other salmonellae. Jpn J Infect Dis. 2008;61(4):313-4.

24. Cocchi S, Grasselli E, Gutacker M, Benagli C, Convert M, Piffaretti JC. Distribution and characterization of integrons in Escherichia coli strains of animal and human origin. FEMS Immunol Med Microbiol. 2007;50(1):126-32.

25. Rehman MU, Zhang H, Huang S, Iqbal MK, Mehmood K, Luo H, et al. Characteristics of Integrons and Associated Gene Cassettes in Antibiotic-Resistant Escherichia coli Isolated from Free-Ranging Food Animals in China. Journal of food science. 2017;82(8):1902-7.

26. Moura A, Soares M, Pereira C, Leitao N, Henriques I, Correia A. INTEGRALL: a database and search engine for integrons, integrases and gene cassettes. Bioinformatics. 2009;25(8):1096-8.

27. Siguier P, Perochon J, Lestrade L, Mahillon J, Chandler M. ISfinder: the reference centre for bacterial insertion sequences. Nucleic acids research. 2006;34(Database issue):D32-6.

28. Kleinheinz KA, Joensen KG, Larsen MV. Applying the ResFinder and VirulenceFinder web-services for easy identification of acquired antibiotic resistance and E. coli virulence genes in bacteriophage and prophage nucleotide sequences. Bacteriophage. 2014;4(1):e27943.

29. Carattoli A, Zankari E, Garcia-Fernandez A, Voldby Larsen M, Lund O, Villa L, et al. In silico detection and typing of plasmids using PlasmidFinder and plasmid multilocus sequence typing. Antimicrob Agents Chemother. 2014;58(7):3895-903.

30. Roberts AP, Chandler M, Courvalin P, Guedon G, Mullany P, Pembroke T, et al. Revised nomenclature for transposable genetic elements. Plasmid. 2008;60(3):167-73.

31. Dong D, Li M, Liu Z, Feng J, Jia N, Zhao H, et al. Characterization of a NDM-1- Encoding Plasmid pHFK418-NDM From a Clinical Proteus mirabilis Isolate Harboring Two Novel Transposons, Tn6624 and Tn6625. Frontiers in microbiology. 2019;10:2030.

32. Sandegren L, Linkevicius M, Lytsy B, Melhus A, Andersson DI. Transfer of an Escherichia coli ST131 multiresistance cassette has created a Klebsiella pneumoniae-specific plasmid associated with a major nosocomial outbreak. J Antimicrob Chemother. 2012;67(1):74-83.

33. Chen YP, Lei CW, Kong LH, Zeng JX, Zhang XZ, Liu BH, et al. Tn6450, a Novel Multidrug Resistance Transposon Characterized in a Proteus mirabilis Isolate from Chicken in China. Antimicrobial agents and chemotherapy. 2018;62(4).

34. de Curraize C, Siebor E, Varin V, Neuwirth C, Hall RM. Two New SGI1-LK Variants Found in Proteus mirabilis and Evolution of the SGI1-HKL Group of Salmonella Genomic Islands. mSphere. 2020;5(2).

35. Gu W, Wang W, Tong P, Liu C, Jia J, Lu C, et al. Comparative genomic analysis of Proteus spp. isolated from tree shrews indicated unexpectedly high genetic diversity. PloS one. 2020;15(2):e0229125.

36. Paitan Y. Current Trends in Antimicrobial Resistance of Escherichia coli. Curr Top Microbiol Immunol. 2018;416:181-211.

37. Choi KY, Spencer JM, Craig NL. The Tn7 transposition regulator TnsC interacts with the transposase subunit TnsB and target selector TnsD. Proc Natl Acad Sci USA. 2014;111(28):E2858-65.

38. Hamilton AL, Kamm MA, Ng SC, Morrison M. Proteus spp. as Putative Gastrointestinal Pathogens. Clinical microbiology reviews. 2018;31(3). 
39. Qu D, Lu S, Wang P, Jiang M, Yi S, Han J. Analysis of CRISPR/Cas system of Proteus and the factors affected the functional mechanism. Life sciences. 2019;231:116531.

40. Martinez N, Rodriguez I, Rodicio R, Mendoza Mdel C, Rodicio Mdel R. Molecular basis and evolution of multiple drug resistance in the foodborne pathogen Salmonella enterica serovar Ohio. Foodborne Pathog Dis. 2010;7(2):189-98.

41. Foley SL, Johnson TJ, Ricke SC, Nayak R, Danzeisen J. Salmonella pathogenicity and host adaptation in chicken-associated serovars. Microbiology molecular biology reviews: MMBR. 2013;77(4):582-607.

42. Munnoch SA, Ward K, Sheridan S, Fitzsimmons GJ, Shadbolt CT, Piispanen JP, et al. A multi-state outbreak of Salmonella Saintpaul in Australia associated with cantaloupe consumption. Epidemiol Infect. 2009;137(3):367-74.

43. Tietze E, Brevet J. The trimethoprim resistance transposon Tn7 contains a cryptic streptothricin resistance gene. Plasmid. 1991;25(3):217-20.

44. Kaushik M, Khare N, Kumar S, Gulati P. High Prevalence of Antibiotic Resistance and Integrons in Escherichia coli Isolated from Urban River Water, India. Microbial drug resistance. 2019;25(3):359-70.

45. Zhang S, Yang H, Rehman MU, Yang K, Dong M, Yang J, et al. Class 1 integrons as predominant carriers in Escherichia coli isolates from waterfowls in Hainan, China. Ecotoxicol Environ Saf. 2019;183:109514.

46. Chen H, Shu W, Chang X, Chen JA, Guo Y, Tan Y. The profile of antibiotics resistance and integrons of extended-spectrum betalactamase producing thermotolerant coliforms isolated from the Yangtze River basin in Chongqing. Environmental pollution. 2010;158(7):2459-64.

47. Lapierre L, San Martin B, Araya-Jordan C, Borie C. Comparison of integron-linked antibiotic resistance genes in strains of Salmonella spp. isolated from swine in Chile in 2005 and 2008. Can J Microbiol. 2010;56(6):515-21.

48. Zhang AN, Li LG, Ma L, Gillings MR, Tiedje JM, Zhang T. Conserved phylogenetic distribution and limited antibiotic resistance of class 1 integrons revealed by assessing the bacterial genome and plasmid collection. Microbiome. 2018;6(1):130.

49. Cleaver SH, Wickstrom E. Transposon Tn7 gene insertion into an evolutionarily conserved human homolog of Escherichia coli attTn7. Gene. 2000;254(1-2):37-44.

50. Flores CC, Cotterill S, Lichtenstein CP. Overproduction of four functionally active proteins, TnsA, B, C, and D, required for Tn7 transposition to its attachment site, attTn7. Plasmid. 1992;28(1):80-5.

51. Deng Y, Bao X, Ji L, Chen L, Liu J, Miao J, et al. Resistance integrons: class 1, 2 and 3 integrons. Ann Clin Microbiol Antimicrob. 2015;14:45.

52. Chen Y, Lei C, Zuo L, Kong L, Kang Z, Zeng J, et al. A novel cfr-carrying Tn7 transposon derivative characterized in Morganella morganii of swine origin in China. J Antimicrob Chemother. 2019;74(3):603-6.

53. Partridge SR. Resistance mechanisms in Enterobacteriaceae. Pathology. 2015;47(3):276-84.

54. Mahillon J, Chandler M. Insertion sequences. Microbiology molecular biology reviews: MMBR. 1998;62(3):725-74.

55. Green B, Bouchier C, Fairhead C, Craig NL, Cormack BP. Insertion site preference of Mu, Tn5, and Tn7 transposons. Mobile DNA. 2012;3(1):3.

56. The Clinical and Laboratory Standards Institute [CLSI]. (2017). Performance Standards for Antimicrobial Susceptibility Testing M02-A12, M07-A10, and M11-A8, 27th Edn. Philadelphia, PA: Clinical and Laboratory Standards Institute, 282.

\section{Figures}


Tn7-like negative $=$ Tn7-like positive

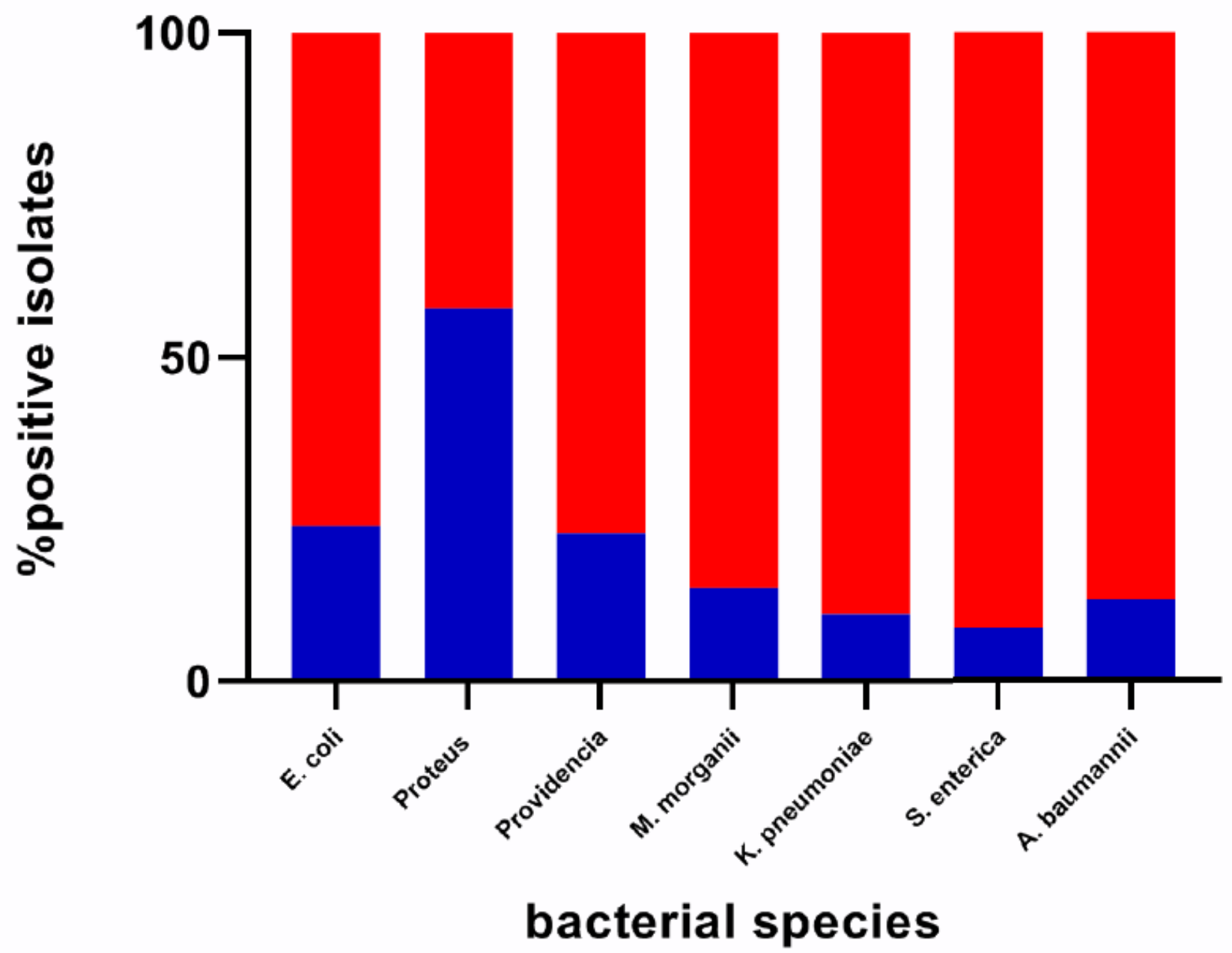

Figure 1

The separation rate of Tn7-like in Enterobacteriaceae of different genera( $n=1482)$. Tn7-like positive; \% of Enterobacteriaceae isolates, which are positive for tnsA gene via PCR, Tn7-like negative; \% of isolates negative for tnsA genes via PCR. Comparison of the incidence of Tn7-like transposons in Enterobacteriaceae isolates versus Tn7-like lacking isolates was assessed using the Chis square test. The results are significant. $p<0.0001<0.05$. 


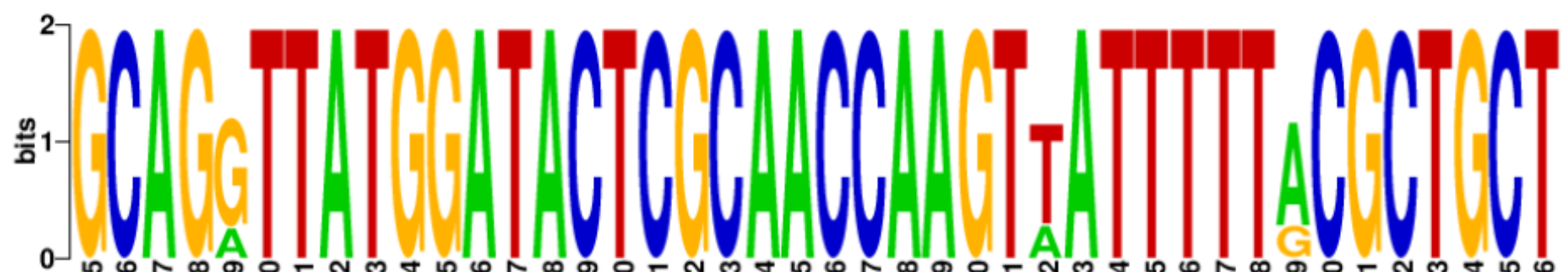

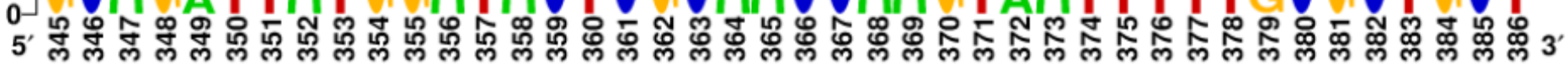
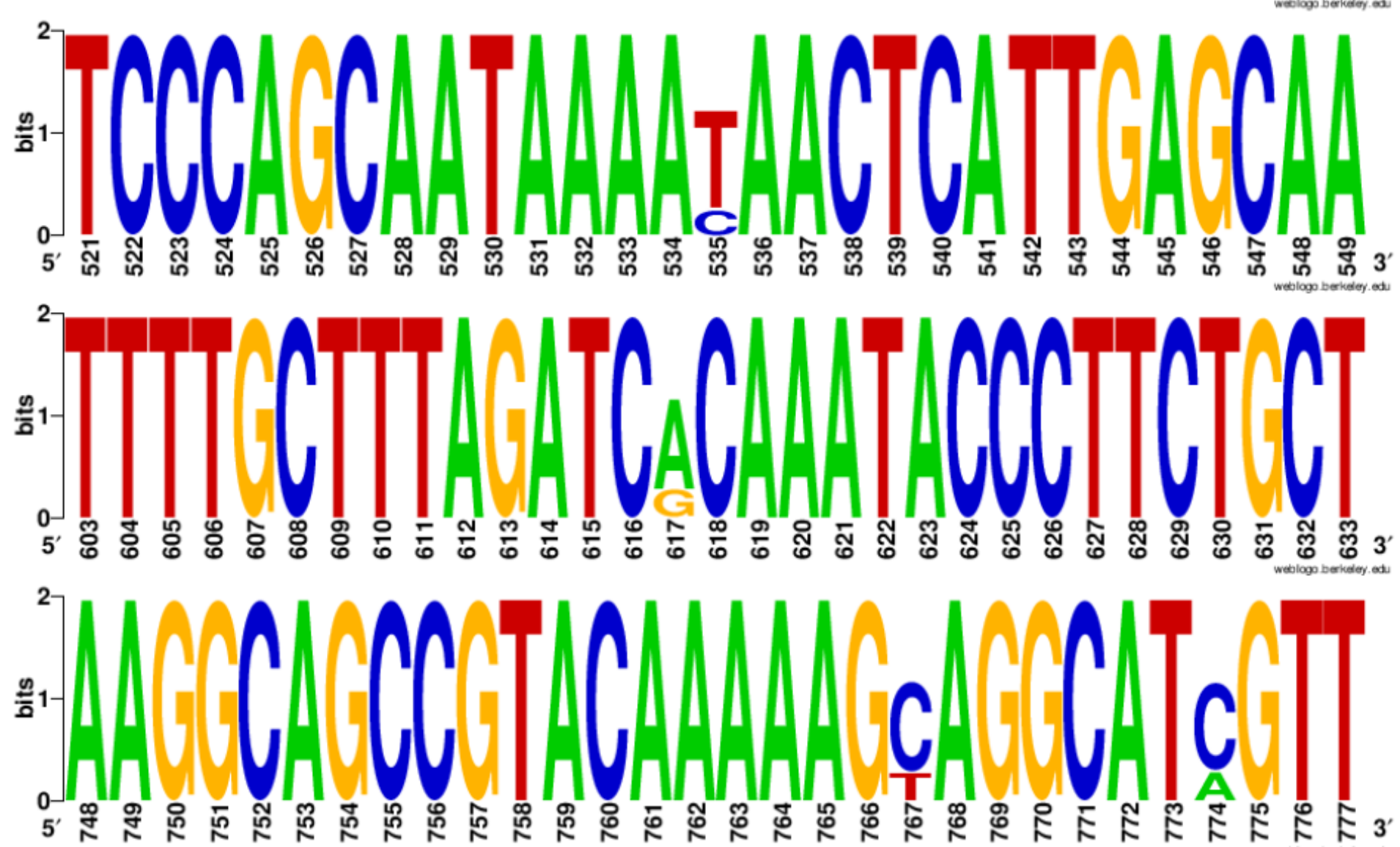

Figure 2

The Weblogo of repeats of int12. The sequence were each mutation site of the integrase gene of class 2 integrons. 


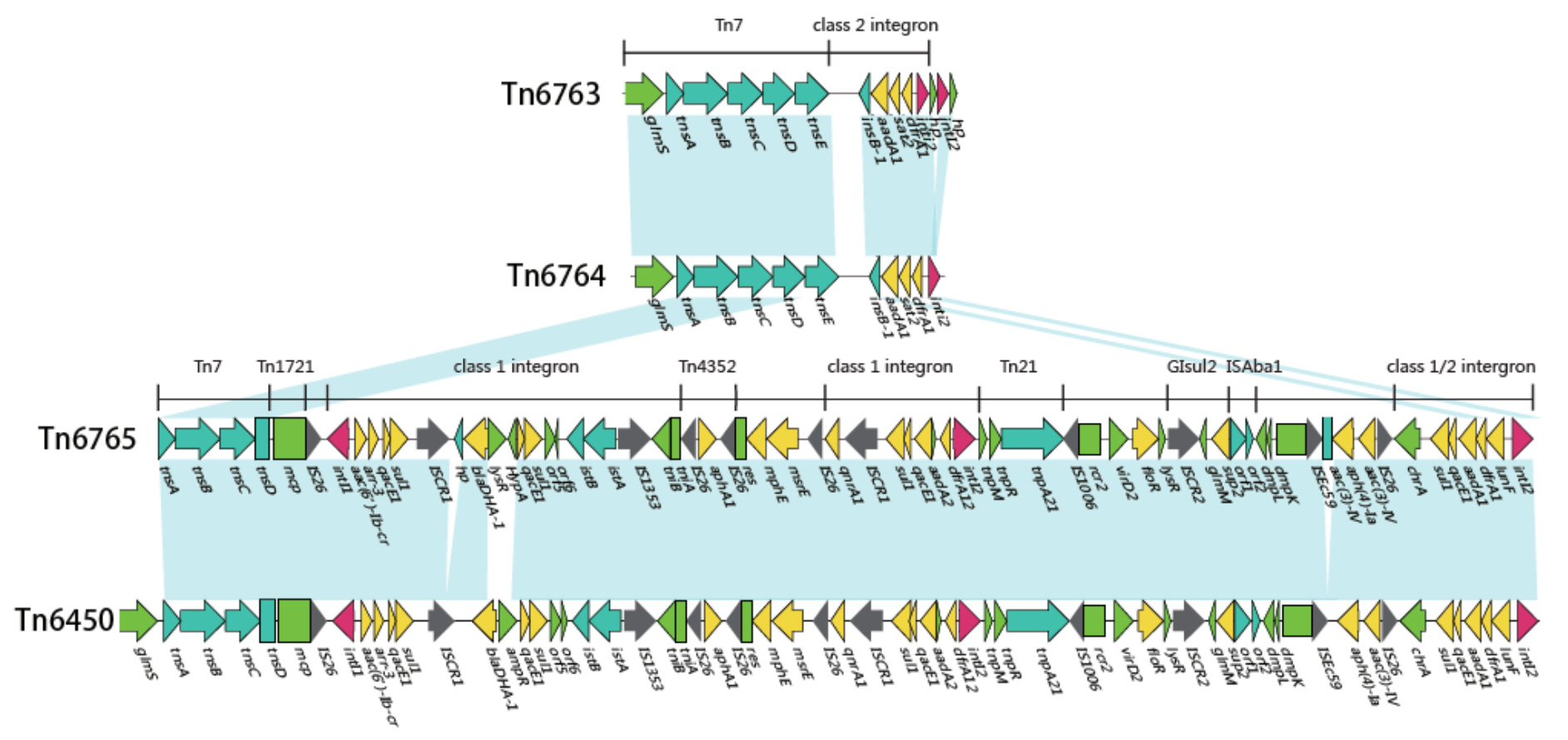

\section{Figure 3}

Genetic structure of Tn6763, Tn6764 and Tn6765. The physical maps were generated using Easyfig 2.2.3 and DNAMAN Version 8.0. Linear comparison of Tn6765 region in p1.1.2 with Tn6763 region in P. mirabilis strain, Tn6764 region in E.coli strain and Tn6450 region in P. mirabilis strain SNYG17 (GenBank accession number MF805806). Genes and ORFs are shown as arrows, and their orientations of transcription are indicated by the arrowheads. Horizontal lines, different regions corresponding to Tn7, Tn1721, Tn21, and integrons. Antimicrobial resistance genes are in yellow. transposase are in blue and integrase genes are in red. The IS elements are indicated by gray arrows. Other functions or putative proteins are in green. Shared regions with $99 \%$ identity are indicated by shading. 


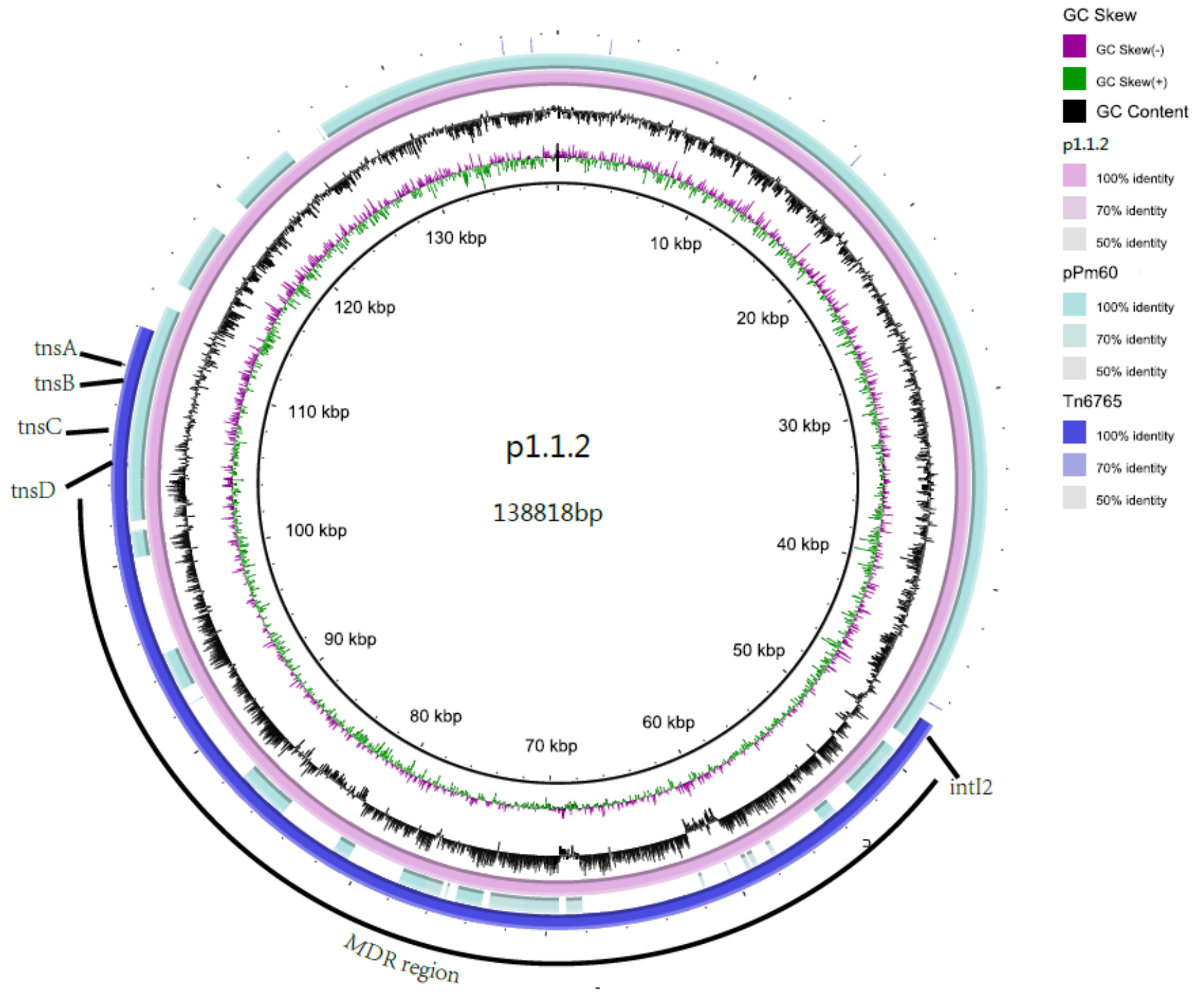

Figure 4

Circular representation of plasmid p1.1.2. The physical map of p1.1.2 was generated using BRIG v0.95. 
P52

CP047287

P1.1.1 CP047113

Tn6451

MG832661
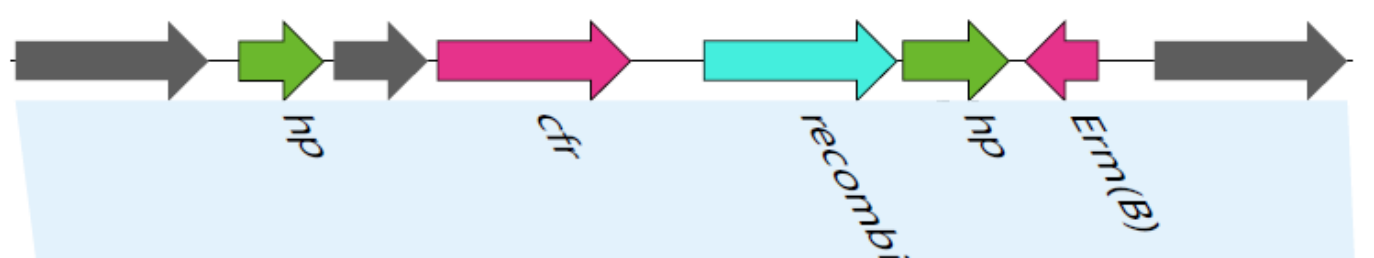

कृ

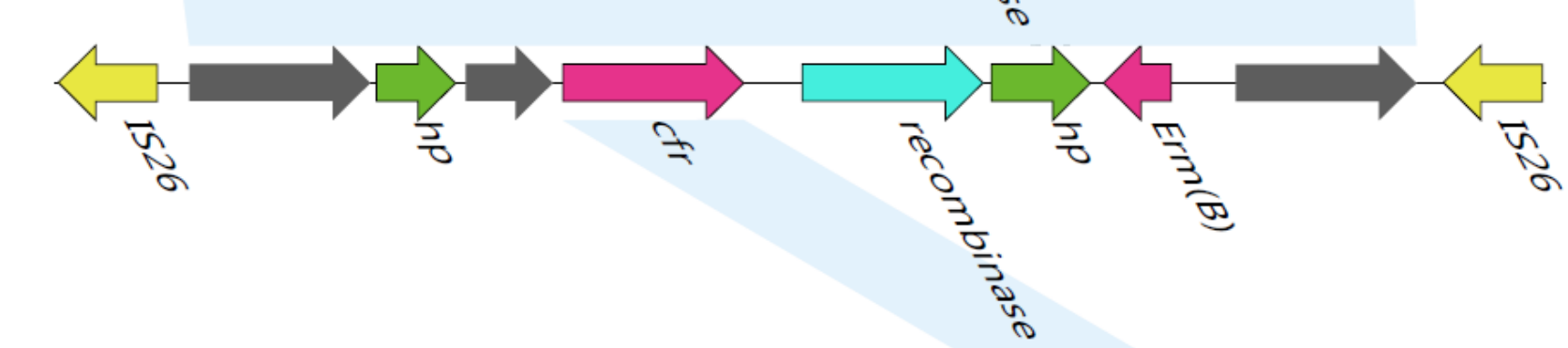

\section{MG832661}

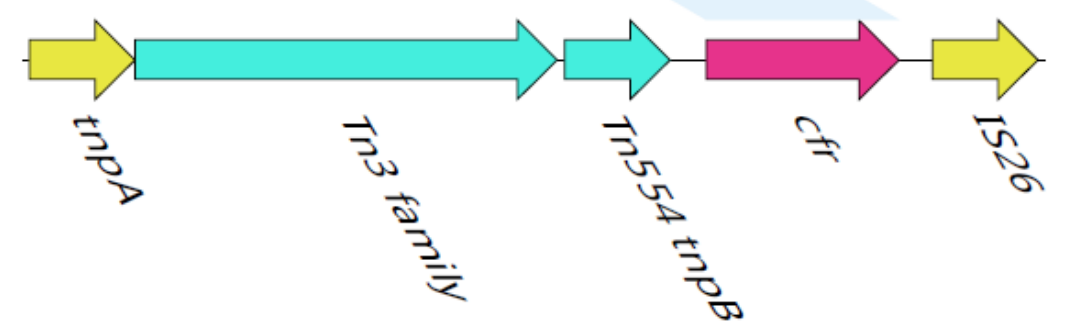

\section{Figure 5}

Genetic structure of cfr-containing region and in p1.1.1. The physical maps were generated using Easyfig 2.2.3 and DNAMAN Version 8.0. Linear comparison of the cfr-containing region in p1.1.1 with the cfr-containing region in P. cibarius strain G11 (Genebank number CP047287) and in M. morganii strain BCMM24 (Genebank number MG832661). Genes and ORFs are shown as arrows, and their orientations of transcription are indicated by the arrowheads. Antimicrobial resistance genes are in red. The IS elements are indicated by yellow arrows. Putative proteins are in green. Shared regions with $99 \%$ identity are indicated by shading. 


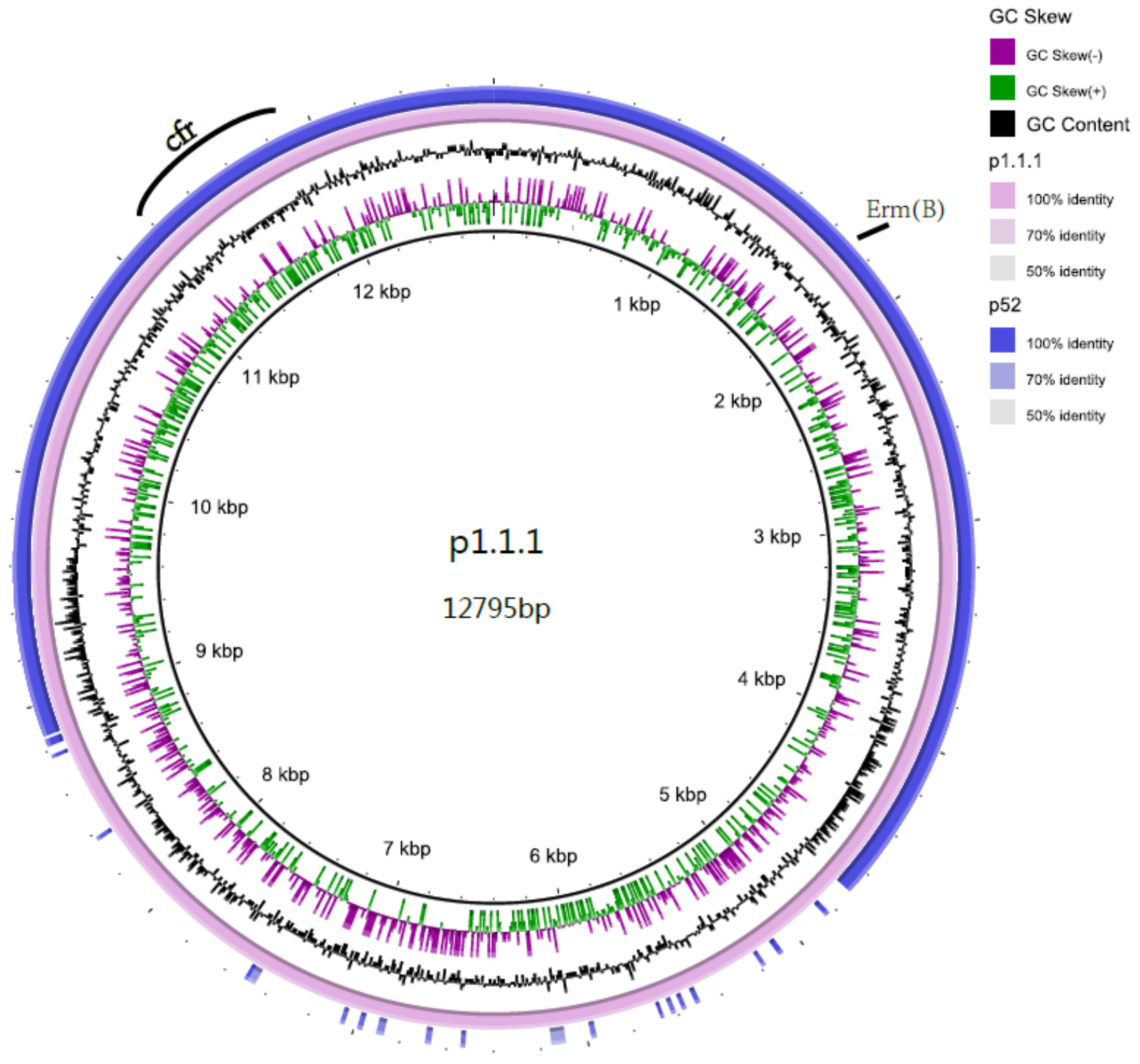

Figure 6

Circular representation of plasmid p1.1.1. The physical map of p1.1.1 was generated using BRIG v0.95.

\section{Supplementary Files}

This is a list of supplementary files associated with this preprint. Click to download.

- Supplementarydata.docx 